\title{
VALORACIÓN DEL IMPACTO DE GÉNERO EN LA SALUD DE MUJERES Y HOMBRES
}

\author{
Pepa Pont Martínez ${ }^{1}$, Cristina Santamaría Navarro ${ }^{2}, M^{a}$ Teofila Vicente Herrero ${ }^{3}$, \\ Montserrat Piñaga Solé M $^{a}$ Victoria Ramírez Iñiguez de la Torre $e^{5} y$ \\ Ángel Arturo López González' \\ pont_pep@gva.es
}

Recibido: 26-02-2010

Aceptado: 16-04-2010

\section{Resumen}

El objetivo es ensayar la utilidad de incorporar variables sensibles al género, fundamentalmente, las relacionadas con la desigual distribución del trabajo productivo y reproductivo, para identificar posibles desigualdades de salud asociadas al género como determinante de salud. Se han realizado entrevistas clínicas, que incluyen exploraciones y un cuestionario complementario con los que se han recogido variables sobre el estado de salud y variables sociodemográficas, incluidas variables sensibles al género. Los datos del cuestionario y la entrevista clínica se han registrado en una base diseñada $a d$ hoc y se ha analizado con el programa SPSS v. 14, aplicando un análisis descriptivo y un análisis de dependencias bivariante.

Palabras clave: género, desigualdades de salud, determinantes de salud.

\footnotetext{
${ }^{1}$ Dirección General de Salud Pública Conselleria de Sanitat. Generalitat Valenciana.

${ }^{2}$ Instituto de Matemáticas Multidisciplinares. Universidad Politécnica de Valencia.

${ }^{3}$ Servicio de Medicina del trabajo de la Dirección General de Correos y Telecomunicaciones. Valencia.

${ }^{4}$ Servicio de Medicina del trabajo del Banco Popular de Valencia.

${ }^{5}$ Servicio de Medicina del trabajo de la Dirección General de Correos y Telecomunicaciones. Albacete

${ }^{6}$ Servicio de Prevención de Riesgos Laborales de GESMA del Gobierno Balear.
} 


\begin{abstract}
The target is to test the utility of incorporating gender sensitive variables, fundamentally the ones related to the unequal distribution of productive and reproductive work, to identify possible health inequalities associated with the gender as determinant of health. Clinical interviews have been carried out, which include explorations and a complementary questionnaire, gathering health state, sociodemographic, and even gender sensitive variables. The questionnaire data and the clinical interview have been registered in a data base designed $a d$ hoc and have been analyzed by the program SPSS v. 14, applying a descriptive analysis and a bivariant analysis of dependencies.
\end{abstract}

Key words: gender, health inequalities, determinants of health.

\title{
1. Introducción
}

Desde el inicio de los años noventa vienen realizándose trabajos que muestran la importancia de los factores ligados al género como determinantes de salud. Sin embargo y a pesar del incremento de la producción científica en diferentes paises (incluyendo España), todavía persiste la carencia de información relacionada con el género (incluso la disponibilidad de datos desagregados por sexo) en la recopilación de datos de los registros sanitarios y documentos clínicos.

Entre los factores diferenciales en relación con el desempeño del género, uno de los aspectos más relevantes, es la desigual distribución del trabajo productivo (remunerado) y reproductivo tal como muestran las encuestas sobre el uso del tiempo (Consejo de Europa. Mainstreaming de género, 1999; Verloo, 2005; Ferrer Pérez, Bosch Fiol y Gili Planas, 1998; Rohlfs, 1999; Vàgerö, 1994; Fuente Sánchez, 2007).

Consideramos fundamental aportar información que contribuya a clarificar la influencia de los aspectos ligados al desempeño del género en la salud de varones y mujeres, especialmente las ligadas a la desigual distribución del trabajo productivo y 
reproductivo. Este trabajo pretende aportar una contribución para incorporar esta perspectiva de género en relación con la salud.

La hipótesis de este trabajo es que la mayor dedicación a las tareas domésticas y de cuidado se asociará a un peor estado de salud.

\section{Objetivos}

El objetivo fundamental de este trabajo es tratar de evidenciar la potencial capacidad explicativa de variables ligadas al desempeño del rol de género y específicamente al desigual reparto del trabajo productivo y reproductivo, como parte fundamental de los factores psicosociales determinantes del estado de salud. Como objetivos específicos nos proponemos:

- Valorar la factibilidad de incluir de manera ordinaria variables sensibles al género que expresen la dedicación a las tareas domésticas y al cuidado, en las entrevistas clínicas y procesos de atención de salud.

- Valorar la capacidad de estas variables sensibles al género para discriminar situaciones diferenciales en la salud de varones y mujeres.

\section{Métodos}

Este trabajo se enmarca en la perspectiva de las desigualdades de género en salud y sesgos de género en atención sanitaria, y por tanto se centra en la identificación de inequidades de salud asociadas a los factores psicosociales relacionados con el desempeño del rol de género, para lo que se incluyen variables que expresan la desigual distribución del trabajo productivo y reproductivo en varones y mujeres. Dentro de esta línea de trabajo en género y salud puede encontrarse el desarrollo de la morbilidad diferencial, es decir, la descripción de la morbilidad por sexo, buscando elementos 
diferenciales que puedan constituir desigualdades de género en salud. En este caso como ya se ha dicho, los elementos diferenciales potencialmente explicativos de las desigualdades de género en salud, se identifican con las variables que dimensionan la dedicación de varones y mujeres a las tareas domésticas y de cuidado.

Entendemos estas variables que tratan de cuantificar la dedicación al trabajo reproductivo, cumplen los requisitos metodológicos de la investigación en ciencias de la salud, tal como define Izabella Rohlfs en sus notas para el diploma de especialización en salud pública y género (2007), es decir, son variables factibles de medir, implican la expresión de resultados que se producen por su mantenimiento en el tiempo, tenemos la oportunidad de obtenerlas y se dispone de sujetos a partir de la oportunidad de captación en los servicios de medicina del trabajo de varios centros, que cuentan con las instalaciones y equipamientos necesarios. Contamos además con la financiación del Plan Nacional de Investigación Científica, Desarrollo e Innovación Tecnológica (I+D+I). Instituto de Salud Carlos III-Subdirección General de Evaluación y Fomento de la Investigación, con el número de referencia de proyecto MD07/00216, dentro de los proyectos complementarios en metodología de la investigación en servicios de salud y equidad social y de género en salud (convocatoria de septiembre de 2007).

Este trabajo recoge así mismo las variables habituales en los exámenes de salud realizados periódicamente desde los servicios de medicina del trabajo en población empleada que voluntariamente acude a las consultas que anualmente son ofertadas para el seguimiento de su estado de salud. Así pues, se recogen también variables procedentes de la exploración y anamnesis, obtenidos en la entrevista clínica, así como variables obtenidos en hemograma y química hemática, además de variables relacionadas con la situación laboral. No obstante, el interés de este artículo se centra en destacar las diferencias en el estado de salud de la población en estudio y el posible valor explicativo de las variables sensibles al género.

Sabemos por diversos estudios que las diferencias y las desigualdades de género, juntamente con la posición socioeconómica son las mayores causas de inequidades en la salud y en la provisión y utilización de los servicios sanitarios (Borrell 
et al., 2004a). Asimismo, existen evidencias de que la incorporación de la perspectiva de género puede reducir las desigualdades en salud y mejorar la eficiencia y efectividad de los servicios (Gender and Health Group-Liverpool Schol of Tropical Medicine, 1998). La importancia del género en la génesis de las desigualdades en la salud deriva de su transversalidad a todos los ámbitos de la salud. El género tiene relación tanto con los aspectos determinantes de la salud y esperanza de vida, las causas de morbimortalidad, las conductas relacionadas con la salud y la disponibilidad, accesibilidad y calidad en la utilización de los servicios sanitarios.

Así pues, este es un estudio de casos, con un muestreo oportunista en población empleada, en el que se incluye la recolección de datos para obtener información de factores psicosociales que incluyen variables sensibles al enfoque de género y específicamente a la desigual distribución del trabajo doméstico y de cuidado en varones y mujeres. Al aprovechar una entrevista clínica ordinaria dentro de las actividades de los servicios de medicina del trabajo, las variables sensibles al género, se obtienen sobre los mismos sujetos y simultáneamente a la información sobre su estado de salud, lo que permite, identificar asociaciones entre la desigual dedicación de varones y mujeres a las tareas domésticas y su estado de salud, estimada a partir de variables clínicas y de laboratorio que son habitualmente manejadas en los procesos de atención.

La captación de la muestra se produjo de manera oportunista a partir de los contactos establecidos desde los servicios médicos ${ }^{7}$ con $1 @$ s trabajador@s que acuden a la revisión médica en 2008 y que voluntariamente aceptan participar en el estudio. Se trata por tanto de población empleada.

La muestra se compone de 793 personas (468 hombres y 325 mujeres). La edad media fue de 44,3 años para los hombres y 40,5 para las mujeres ${ }^{8}$. Para obtener los

\footnotetext{
${ }^{7}$ En tres servicios médicos de empresa en Valencia y Albacete (Servicio de Medicina del trabajo del Banco Popular de Valencia, Servicio de Medicina del trabajo de la Dirección General de Correos y Telecomunicaciones. Valencia y Servicio de Medicina del trabajo de la Dirección General de Correos y Telecomunicaciones. Albacete).

${ }^{8}$ Esta diferencia es estadísticamente significativa.
} 
datos se diseñó un cuestionario ad-hoc en el que se recogía información acerca de las características sociodemográficas, que incluía la dedicación al trabajo productivo, tareas domésticas y de cuidado. El cuestionario también recoge las condiciones de salud, mediante anamnesis y exploración, (incluyendo declaración de la autopercepción de salud) y análisis de sangre (hematíes, $\mathrm{Hb}$, hematocrito, fórmula leucocitaria, Fe sérico, colesterol (total y fracción HDL + LDL), triglicéridos urea, creatinina, GOT, GPT, GGT, Proteína C reactiva). Aunque el cuestionario se diseñó pensando que fuera autoadministrado, finalmente se cumplimentó mediante entrevista, dentro de la visita a los servicios médicos, como complemento a las revisiones de salud.

Los datos obtenidos a partir de la entrevista clínica y el cuestionario, así como los resultados analíticos, se han registrado en una base de datos específicamente diseñada para el estudio y han sido analizados con el programa estadístico SPSSV 14, aplicando un análisis descriptivo y un análisis de dependencias (análisis bivariante). Los resultados se presentan como medias y desviación estándar o porcentajes. Usamos un test de t de Student o la U de Mann-Whitney, para variables cuantitativas, y la prueba de Chi-cuadrado para variables cualitativas Se consideró un nivel de significación de las diferencias con un valor de $\mathrm{p}<0.05$.

\section{Fortalezas y debilidades}

Consideramos que una de las riquezas de este trabajo es precisamente la obtención de variables clínicas ordinarias en los procesos de atención y de datos sociodemográficos que incluyen variables sensibles al género de los mismos sujetos de estudio, lo que es muy difícil de obtener en los registros sanitarios habituales y por tanto una de las causas de las dificultades para encontrar hallazgos significativos en relación con la influencia del género en la salud.

Asumimos que como señala Naila Caber, reducir el análisis de género sólo a aspectos referidos a la división sexual y social del trabajo, no permite visualizar en toda su 
complejidad las dimensiones culturales, políticas y personales de las relaciones de género, sin embargo, consideramos que aún con estas limitaciones, esta aportación puede implicar una sustancial mejoría en el conocimiento del impacto del género en la salud.

La cantidad de variables que debe manejarse y el tamaño muestral, no han permitido encontrar diferencias significativas en subgrupos muestrales que podrían haber manifestado, confirmado (o refutado) de manera más completa la hipótesis inicial.

Consideramos también que sería necesario profundizar en la recogida homogénea y sistemática de la información, de modo que los resultados obtenidos para las distintas variables constituyan un método lo más fiable preciso y homogéneo para todos los sujetos a estudio. La variabilidad de centros y profesionales implicados, que por una parte puede ser una riqueza del estudio, puede por otro lado constituir una cierta debilidad metodológica.

\section{Resultados y Discusión}

En este estudio realizado sobre 468 hombres y 325 mujeres no existe diferencia significativa entre varones y mujeres en el nivel de formación, personas con las que se convive, número y edades de hijos e hijas, tipo de empresa. Los varones disponen de más tiempo de ocio que las mujeres pero esta diferencia no alcanza significación estadística. Si hay significación estadística para la dedicación a la actividad deportiva, a la que los varones dedican más tiempo que las mujeres (27,52 minutos diarios de media para varones frente a 22,79 minutos de las mujeres).

Respecto a las condiciones diferenciales en el trabajo productivo de hombres y mujeres, encontramos diferencias estadísticamente significativas en la responsabilidad laboral desempeñada por varones y mujeres, de manera que las mujeres ocupan en mayor proporción los puestos bases, mientras los hombres ocupan significativamente más, puestos intermedios y de jefatura. También encontramos diferencias significativas en función del sexo, para la situación laboral de manera que los hombres tienen 
contratos fijos en mayor proporción $(90,6 \%$ vs $74,2 \%$ ) y las mujeres son más frecuentemente eventuales (el 65,6\% vs 34,4\%). Calculando la OR, podemos decir que el porcentaje de varones con contrato fijo es más de tres veces mayor que el de mujeres. En coherencia con las diferencias en el tipo de puestos desempeñados por varones y mujeres, existen diferencias significativas en el salario, que es significativamente mayor en varones. También la antigüedad laboral es mayor en los varones (los varones tienen una vida laboral media de 22,43 años vs 15,14 años para las mujeres).

En cuanto a las condiciones diferenciales en el trabajo reproductivo de hombres y mujeres observamos diferencia estadísticamente significativa en el tiempo dedicado al cuidado de las personas dependientes (88,05 minutos al día para las mujeres vs 55,04 minutos diarios para los varones, es decir las mujeres dedican 1,6 veces más tiempo que los varones). También hay diferencias significativas en el tiempo dedicado a la limpieza y el orden en el hogar (con una media de dedicación de 111,53 minutos para las mujeres vs 60,97 para los hombres, es decir las mujeres dedican 1,8 veces más tiempo que los varones).

Aunque las diferencias son menores, también son las mujeres las que dedican un tiempo significativamente mayor a las gestiones y las compras para el hogar (con una media de 39,6 minutos diarios frente a los 35,05 minutos diarios dedicados por los varones).

Sobre las condiciones diferenciales en el estado de salud, al contrario de lo que han encontrado muchos otros estudios, en nuestro trabajo no se encuentran diferencias significativas en la percepción de salud para varones y mujeres. Tampoco existen diferencias significativas en el consumo de tabaco, aunque hay mayor proporción de mujeres fumadoras (fuman el 34,5\% de las mujeres vs $29,9 \%$ de los varones). Para el consumo de alcohol, los varones son significativamente más bebedores (sólo un 19,4 se declaran no bebedores, frente al 40,9\% de las mujeres, mientras que casi el $12 \%$ de los varones frente al $1 \%$ de las mujeres declaran beber habitualmente).

Aunque hay diferencias en las cifras tensionales para varones y mujeres, las medias de tensión arterial son normales para unos y otras. Hay diferencias 
estadísticamente significativas para el IMC, de manera que los varones tienen un IMC medio de 27,24 y las mujeres 24,7. A pesar de que un 51,4\% de la muestra tiene valores alterados de colesterol total, la media está dentro del rango de normalidad. La media de colesterol total es ligeramente superior a lo normal en los varones (media de 204 $\mathrm{mg} / \mathrm{ml}$, sin significación clínica). Las demás cifras del hemograma y bioquímica sanguínea, tienen medias dentro de los rangos aceptados como normales, tanto en varones como en mujeres.

Pensamos que la similitud en la valoración de la percepción de salud en hombres y mujeres en nuestro estudio, puede deberse a la baja proporción de respuestas obtenidas en este apartado del cuestionario y a la diferente edad media para unos y otras. Una vez ajustada por edad, para mayores de 41 años, tienen una percepción de salud significativamente mejor las personas con salarios por encima de $18.000 €$. No observamos diferencias en la percepción de salud en función del tiempo dedicado a tareas domésticas ni de cuidados (ni en hombres ni en mujeres).

Para menores de 40 años (hombres y mujeres conjuntamente) tienen una proporción significativamente mayor de HTA, quienes dedican más de 116 minutos al día a las tareas domésticas y al cuidado. Por otro lado las personas mayores de 40 años (hombres y mujeres conjuntamente) que dedican más de 116 minutos diarios a tareas domésticas y de cuidado, tienen tasas significativamente más altas de consumo de tabaco.

\section{Conclusiones}

A pesar de que no encontramos datos suficientes para validar nuestra hipótesis inicial, los hallazgos encontrados parecen sugerir que en efecto la mayor dedicación al trabajo reproductivo se asocia a incrementos en al menos algunos factores de riesgo para la salud. 
Considerando la peor posición laboral de las mujeres y sus menores salarios, es probable que se beneficien menos del factor protector para la HTA que implica contar con salarios mayores de $18.000 €$.

Considerando que las mujeres tienen una mayor dedicación a las tareas domésticas y de cuidado, es probable que esto constituya un factor de riesgo para la HTA más importante para las mujeres que para los hombres.

Consideramos que la falta de hallazgos más precisos del impacto diferencial de la dedicación a las tareas domésticas y de cuidado sobre la salud de hombres y mujeres puede deberse en parte a las debilidades metodológicas ya señaladas y en parte a las diferencias en las medias de edad de hombres y mujeres.

A pesar de las limitaciones del estudio, se pone de manifiesto la factibilidad de obtener datos que aporten información relacionada con el género en los procesos de atención de salud, sin que esto represente una sobrecarga inaceptable.

Registrar variables sensibles al género (al menos a la desigual distribución del trabajo productivo y reproductivo) permite identificar algunos de los efectos de las diferencias de género en la salud.

Creemos que es necesario seguir profundizando en este tipo de trabajos.

Nos parece imprescindible aportar datos que faciliten y animen nuevas y mejores investigaciones (tanto cuantitativas como cualitativas) en salud y género y en la medida de lo posible, introducir aspectos cada vez más complejos pero también más acertados, no sólo desde la perspectiva de las desigualdades de salud, sino también sobre el papel del género como determinante de salud.

\section{Agradecimientos}

Agradecemos su disponibilidad a las personas que han aceptado aportar información para este estudio. 


\section{BIBLIOGRAFÍA}

- Artatcoz, L; Cortes I.; Moncada S.; Rohfs I. y Borrell, C. (1999): "Diferencias de género en la influencia del trabajo doméstico sobre la salud” En: Gaceta Sanitaria, vol. 13, no. 3, pp. 201-7.

- Artazcoz, L. et al. (2007): "Los determinantes de la salud en una perspectiva integradora de los enfoques de género, clase social y trabajo”. En C. Borrell y L. Artazcoz (coords.): Investigación en género y salud. Sociedad Española de Epidemiología, monografía no. 5, pp 21-43.

- Borrell C; Garcia-Calvente M. M. y Marti-Bosca J. V. (2004a): "La salud pública desde la perspectiva de género y clase social”. En Gaceta Sanitaria, may, vol.18, Supl. 1, pp 2-6.

- Borrell C.; Rohlfs, I.; Artazcoz, L. y Muntaner, C. (2004b): “Desigualdades en salud según la clase social en las mujeres. ¿Cómo influye el tipo de medida de la clase social?”. En Gaceta Sanitaria, vol. 18, pp 75-82.

- Consejo de Europa, (1999): "Mainstreaming de género. Marco conceptual, metodología y presentación de "buenas prácticas". Informe final de las actividades del Grupo de especialistas en mainstreaming (EG-S-MS), (versión español e inglés), Serie documentos, $\mathrm{n}^{\circ}$. 28. Madrid: Instituto de la Mujer.

- Durán de las Heras, M. A. (2004): “Las demandas sanitarias de las familias". En Gaceta Sanitaria, vol. 18, supl. 1, pp. 195-200.

- Ferrer Pérez, V. A.; Bosch Fiol, E. y Gili Planas, M. (1998): “Aspectos diferenciales en salud entre mujeres que trabajan fuera del hogar y las amas de casa". En Psicothema, vol. 10, no. 1, pp. 53-63.

- Fuente Sánchez, M. (2007): Usos del tiempo, estereotipos, valores y actitudes. Madrid: Instituto de la Mujer.

- Garrido Luque, A. (2000): "El reparto del trabajo no remunerado: Expectativas y deseos de cambio". En Cuaderno de Relaciones Laborales, nº 17, pp. 15-38.

- Gender and health group-Liverpool school of tropical medicine (1998): Guidelines for the analysis of Gender and Health. Liverpool: University of Liverpool. 
- Kabeer, N. (1998): Realidades trastocadas. Las jerarquías de género en el pensamiento del desarrollo. México, Paidós.

- Macintyre, S.; Hunt, K. y Sweeting, H. (1996): “Gender differences in health: are things really as simple as they seem?” En Social Science \& Medicine, vol. 42, nº 4, pp. 617-624.

- Rohlfs, I. y Frigola, M. (2007): “La incorporación de la perspectiva de género en las encuestas de salud de los primeros años del siglo XXI: evolución y camino a seguir". En Borrell, C. y Artazcoz, L. (coords.): Investigación en género y Salud. Sociedad Española de Epidemiología, monografía no. 5, pp. 44-78.

- Ruiz, M. T. y Verbrugge, L. M. (1997): “A two way view of gender bias in medicine". En Journal Epidemiol Community Health, vol. 51, nº. 2, pp 106-109.

- Vàgerö D. (1994): “¿Ha contribuido la creciente participación de la mujer en el mercado laboral a mejorar su salud o no? Análisis de la mortalidad femenina". En Quadern CAPS, Primavera, $\mathrm{n}^{\circ} 21$, pp. 17-23.

- Valls-Llobet, C. (2006): Mujeres invisibles. Barcelona: De Bolsillo.

- Velasco Arias, S. (2006): Atención biopsicosocial al malestar de las mujeres. Madrid: Instituto de la Mujer. Secretaría General de Políticas de Igualdad. Ministerio de Trabajo y Asuntos Sociales.

. (2008): Recomendaciones para la práctica del enfoque de género en programas de salud. Madrid: Observatorio de la Salud de la Mujer. . (2009): Sexos, género y salud. Teoría y métodos para la práctica clínica y programas de salud. Madrid: Minerva.

- Verloo, M. (2005): "Mainstreaming Gender Equality in Europe. A Frame Analysis Approach". En The Greek Review of Social Research (Special issue: Differences in the Framing of Gender Inequality as a Policy Problem Across Europe), 117 B, pp. 11-34.

- Vlassoff, C. y Garcia Moreno, C. (2002): "Placing gender at the centre of health programming: challenges and limitations”. En Social Science \& Medicine, jun, vol. 54, $\mathrm{n}^{\mathrm{o}} .11$, pp. 1713-23. 